\title{
HPV vaccines: peering through the fog
}

\section{Anne Szarewski}

\section{Background}

The UK's human papillomavirus (HPV) vaccination programme should now be underway: vaccination of 12-13year-old girls against HPV was due to begin in September 2008, with a recent announcement that 17-18-year-olds will also be vaccinated in this academic year. ${ }^{1}$ From September 2009, a further catch-up programme for girls up to the age of 18 years will commence. The Department of Health (DH) has decided that the HPV vaccine to be used for the programme will be Cervarix ${ }^{\circledR}$ (GlaxoSmithKline), which is the bivalent vaccine against the cervical cancer-causing HPV types 16 and 18 . This decision has surprised many, in view of the fact that the other available vaccine, Gardasil ${ }^{\circledR}$ (Merck), would protect against genital warts (HPV types 6 and 11) as well as cervical cancer.

It is unfortunate that there has not been a proper disclosure of the factors that led to the decision, resulting in widespread speculation amongst health professionals and the media, particularly relating to the cost of the vaccine. A list of the pre-agreed evaluation criteria against which the bids were evaluated has recently been released (Table 1), and includes many factors, which have been weighted, though details of cost are still not given. When questioned, the Secretary of State for Health stated that both published and unpublished data were considered. ${ }^{2}$ The suggestion in the media that girls in the UK will receive an inferior vaccine may undermine the success of the vaccination programme, as parents become unsure whether it is worth vaccinating their daughter with such a product. ${ }^{3,4}$ Adding fuel to this debate is a mathematical model published in the $B M J$, which was considered by the DH when making its decision. ${ }^{5}$ This suggests that Cervarix must be $£ 13-£ 21$ cheaper than Gardasil in order to be cost effective. However, it should be noted that a number of assumptions were made in this model; it was assumed both vaccines were identical in terms of efficacy, duration of immunity, cross-protection against nonvaccine HPV types, and that uptake amongst girls would be at least $80 \%$. This last assumption is the least likely to be correct; a pilot study in a primary care trust (PCT) in Manchester found uptake was only $70 \%$ overall, and significantly lower in schools with a greater proportion of girls from ethnic minority groups and poorer families. In addition, the Catholic schools in the PCT refused to participate in the pilot. ${ }^{6}$ One could speculate that another factor influencing the $\mathrm{DH}$ decision might have been the possibility of greater acceptability of a 'cervical cancer vaccine' (without the need to mention genital warts) in certain ethnic and religious groups. ${ }^{7}$

\section{Age for vaccination}

For optimal protection, the vaccines should be

J Fam Plann Reprod Health Care 2008; 34(4): 207-209

Cancer Research UK Centre for Epidemiology, Mathematics and Statistics, Wolfson Institute of Preventive Medicine, London, UK

Anne Szarewski, PhD, FFSRH, Clinical Consultant and Honorary Senior Lecturer, and Editor-in-Chief, Journal of Family Planning and Reproductive Health Care

Correspondence to: Dr Anne Szarewksi, Cancer Research UK Centre for Epidemiology, Mathematics and Statistics, Wolfson Institute of Preventive Medicine, Charterhouse Square, London EC1M 6BQ, UK. E-mail: anne.szarewski@cancer.org.uk
Table 1 Pre-agreed award criteria for the evaluation of the contract to supply human papillomavirus (HPV) vaccine ${ }^{2}$

\begin{tabular}{ll}
\hline Criteria & $\begin{array}{l}\text { Points } \\
\text { (maximum) }\end{array}$ \\
\hline $\begin{array}{l}\text { Quality of protection against cervical cancers } \\
\text { caused by HPV strains 16/18 }\end{array}$ & 5000 \\
$\begin{array}{l}\text { Duration of protection against cervical cancers } \\
\text { caused by HPV strains 16/18 for more than 10 }\end{array}$ & 3000 \\
$\begin{array}{l}\text { years' duration } \\
\begin{array}{l}\text { Quality of protection against anogenital warts } \\
\text { caused by HPV strains 6/11 }\end{array}\end{array}$ & 1300 \\
\hline
\end{tabular}

Duration of protection against anogenital warts 500 caused by HPV strains $6 / 11$ for more than 10 years' duration

Quality of protection against HPV strains not included in the vaccine formulation

Other evidence of additional clinical benefits 500

Effective price per dose excluding VAT

Supply of the vaccine as single pre-filled 10 syringe pack presentation

Quality of labelling, leaflets and presentation 5

Shelf-life

Flexibility in the vaccine dosage schedule

Offers that reduce the risk of wastage if the vaccine is subject to temperatures above $8^{\circ} \mathrm{C}^{a}$

Closeness of proposed delivery schedule to 200 authority requirements

Pallet configuration including a preference for the use of Euro pallets

Impact of proposed amendments on the terms and conditions

$(-500)$ [Offerors may lose up to 500 points]

Quality/robustness of manufacturing 10 contingency arrangements

Quality/robustness of the risk management of storage and distribution

Information provided relating to pack sizes, cold chain delivery, batch numbering systems and production capacity

alncludes the provision of temperature indicators and evidencebased guidance on the stability of the vaccines at higher storage temperatures and subsequent safe administration.

administered prior to the onset of sexual activity. In general, antibody responses induced by vaccines are higher pre-puberty compared to post-puberty, a feature that has also been shown for the HPV vaccines. Currently followup data are available only up to 6.4 years, but modelling suggests that immunity may persist for 30 years. ${ }^{8}$ Ideally, such a vaccine would be administered with other childhood vaccines, removing any link with sexual activity in the minds of parents.

\section{The vaccines}

Both Gardasil and Cervarix have shown high efficacy, approaching 100\%, against HPV 16 and 18-related highgrade cervical intraepithelial neoplasia (CIN) and Gardasil has shown similar efficacy against genital warts. ${ }^{8}$ Although 
superficially similar, there are differences between the two vaccines, which may turn out to be relevant. Both consist of virus-like particles (VLPs) using the L1 virus coat proteins. VLPs have the outward appearance of the actual virus and generate a powerful immune response (much stronger than natural immunity) but are harmless as they contain no DNA.

The VLPs in the two vaccines have been manufactured using different systems, and the adjuvants used are also different. While Gardasil contains aluminium hydroxyphosphate sulphate, Cervarix is adjuvanted with ASO4, which is aluminium hydroxide with monophosphoryl lipid A. The ASO4 adjuvant has been used previously in a hepatitis B vaccine (Fendrix ${ }^{\circledR}$ ), where it was shown to generate a stronger and longer-lasting immune response than the vaccine containing aluminium hydroxide alone. ${ }^{9}$ In this context, it is interesting that the antibody response to HPV 18 appears to be maintained at high levels for longer with Cervarix than Gardasil ${ }^{9}$ though the clinical significance of this is still uncertain. One could speculate that higher antibody levels might translate into longer lasting immunity, a criterion weighted heavily in the UK evaluation (Table 1) and in a recent economic evaluation in the USA. ${ }^{10}$ Clearly, if one vaccine required a booster after a certain length of time while the other did not, the difference in costs to a vaccination programme would be very significant. A direct comparison study of the two vaccines is underway, using a non-proprietary neutralising antibody assay; interim results should be available before the end of this year and are awaited with interest.

\section{Cross-protection}

A related issue is that of cross-protection. It had been thought unlikely that this would occur, yet both vaccines have shown early evidence of such an effect. In the extended Phase II trials at 6.4 years, Cervarix has shown $78 \%$ (95\% CI 39-93) protection against incident infections with HPV 45, which is HPV 18-related, and $60 \%$ (95\% CI 20-81) protection against incident infections with HPV 31, which is HPV 16-related. ${ }^{11}$ In the interim analysis of the Phase III trial, Cervarix has shown cross-protection against persistent infections at 6 months for HPV type $45(59.9 \%, 95 \%$ CI 2.6-85.2), type 31 (36.1\%, 95\% CI 0.5-59.5) and type $52(31.6 \%, 95 \%$ CI 3.5-51.9). The extent of sustained cross-protection against persistent infections, abnormal cytology and precancerous lesions remains to be determined. A laboratory study of antibody cross-neutralisation with Gardasil suggested cross-protection against types 31, 45, 52 and 58,8 while more recently data have been presented suggesting protection against both persistent HPV infection and CIN associated with a bank (31/33/45/52/58) of non-vaccine HPV types. ${ }^{12}$ However, interestingly, in the Canadian Summary of Product Characteristics, dated March 2008, the level of protection against individual HPV types is given; here there is no cross-protection against HPV type 45.13 Although the clinical significance of this is not yet known, it is interesting that there appears to be a consistently weaker effect of Gardasil on HPV 18 and its related type, 45 (see above). Cross-protection is an important issue, as it may raise the overall protection level significantly.

\section{Other issues}

\section{Vaccinating older women}

Studies are underway to evaluate the benefit of vaccinating older women (i.e. those over 25 years of age). In a group of previously HPV-negative women aged 24-45 years, recently presented data suggest that Gardasil was highly effective in preventing both persistent HPV infection and disease associated with the HPV types in the vaccine. ${ }^{14}$ If efficacy in older women is confirmed, vaccination of a wider age range could have a more immediate impact on cervical cancer.

\section{Need for third vaccine dose?}

Is the third dose of vaccine actually necessary? A threedose schedule is complicated and particularly problematic in developing countries. Could one 'mix and match' the two vaccines to try and reap the benefits of both? We do not have answers to these questions at present but they are urgently needed.

\section{Future of cervical screening?}

In theory, an HPV vaccine could prevent almost all cervical cancer, eventually removing the need for cervical smears. It is noteworthy that the vaccines should be effective against cervical adenocarcinoma (of which a relatively higher proportion are associated with HPV 18 and 45), which is not detected effectively in current screening programmes and which appears to be increasing in incidence. There is potential for a very significant reduction in this cancer, which now accounts for up to $20 \%$ of cervical cancers. However, until the number of HPV types in the vaccine is increased, there will still be cancers not prevented by vaccination. In addition, there is at least one whole generation of women for whom the vaccines have come too late to precede sexual activity, and who will continue to require screening. It is, however, clear that screening programmes, where they exist, will need to adapt when HPV vaccination becomes widespread. It is likely that cytology will become less useful as the number of abnormalities in the population decreases, and cytologists struggle to maintain their skills. HPV testing of some kind is likely to be the way forward, but this raises many issues - not least the high transient positivity rates in women under 35 years, and the probable need for HPV typing tests, which are not yet commercially available, to monitor longterm vaccine efficacy. These are issues that will need to be addressed in the next 15 years in the UK.

\section{Vaccinating boys}

Data on efficacy in men are awaited, as the issue of whether to vaccinate boys as well as girls is still under debate. Most mathematical models suggest that vaccination of girls alone is the most cost-effective strategy, assuming high uptake amongst girls. However, if boys are not vaccinated, men who have sex with men (MSM), and who are at increased risk of HPV infections and anal cancer, will not benefit from the vaccine. Another unfortunate aspect of restricting vaccination to girls is that it focuses attention on women in relation to a sexually transmitted virus; this is not a useful social message in any context and there are some cultures in which the strategy may prove unacceptable.

Assuming efficacy in men is shown, I suggest that an ideal solution would be to vaccinate girls with Cervarix and boys with Gardasil. Evidence is accumulating to suggest that Cervarix may offer greater protection against cervical cancer, which would make it the more logical choice for girls. If boys were vaccinated with Gardasil they would improve herd immunity against the cancer-causing HPV types, while also being protected themselves (a particular benefit for MSM). Meanwhile, their vaccination against HPV types 6 and 11 would, again via herd immunity, protect both sexes. Finally, both sexes would be seen as susceptible, which, in my view, would be a far more equitable public health message. 
Statements on funding and competing interests

Funding The author has received honoraria and conference sponsorship from GlaxoSmithKline and Sanofi-Pasteur.

Competing interests The author is an investigator in the Cervarix trials. She is also Editor-in-Chief of the Journal of Family Planning and Reproductive Health Care.

\section{References}

1 Salisbury D. Introduction of HPV vaccination into the national immunisation programme: vaccination of 17- to 18-year-old young women in 2008/09. DH letter GW 10266, 22 July 2008. http://www.immunisation.nhs.uk/publications/HPV_DSletter220 708.pdf [Accessed 22 August 2008].

2 Hansard, Columns 531W, 532W, 16 July 2008. http://www. publications.parliament.uk/pa/cm200708/cmhansrd/cm080716 /text/80716w0028.htm [Accessed 22 August 2008].

3 Hawkes N. GlaxoSmithKline wins NHS contract to supply cervical cancer vaccine. The Times, 18 June 2008 http://www.timesonline.co.uk/tol/life_and_style/health/article41 66423.ece [Accessed 22 August 2008].

4 Boseley S. Decision on cervical cancer vaccine is false economy, say health groups. The Guardian, 18 July 2008. http://www.guardian.co.uk/science/2008/jul/18/cancer.medicalr esearch [Accessed 22 August 2008].

5 Jit M, Choi $\mathrm{YH}$, Edmunds WJ. Economic evaluation of human papillomavirus vaccination in the United Kingdom. BMJ 2008; 337: a769.

6 Brabin L, Roberts SA, Stretch R, Baxter D, Chambers G, Kitchener $\mathrm{H}$, et al. Uptake of first two doses of human papillomavirus vaccine by adolescent schoolgirls in Manchester: prospective cohort study. BMJ 2008; 336 : 1056-1058.

7 Kane MA. Human papillomaviruses (HPV) vaccines: implementation and communication issues $J$ Fam Plann Reprod Health Care 2008; 34: 3-4.
8 Stanley M. HPV vaccines: where are we now? J Fam Plann Reprod Health Care 2007; 33: 227-229.

9 Einstein $\mathrm{MH}$. Acquired immune response to oncogenic human papillomavirus associated with prophylactic cervical cancer vaccines. Cancer Immunol Immunother 2008; 57: 443-451.

$10 \mathrm{Kim}$ JJ, Goldie SJ. Health and economic implications of HPV vaccination in the United States. $N$ Engl J Med 2008; 359: 821-832.

11 Harper D, Gall S, Naud P, Quint W, Dubin G, Jenkins D, et al. Sustained immunogenicity and high efficacy against HPV16/18 related cervical neoplasia: long-term follow-up through 6.4 years in women vaccinated with CERVARIX (GSK's HPV $16 / 18$ AS04 candidate vaccine). Oral presentation at the Society of Gynecologic Oncologists Annual Meeting on Women's Cancer, Tampa, FL, USA, 10 March 2008 [Abstract in Gynecol Oncol 2008; 109: 158]

12 Brown D. HPV Type 6/11/16/18 vaccine: first analysis of crossprotection against persistent infection, cervical intraepithelial neoplasia (CIN), and adenocarcinoma in situ (AIS) caused by oncogenic HPV types in addition to 16/18. Oral presentation at the 47th Interscience Conference on Antimicrobial Agents and Chemotherapy (ICAAC), Chicago, IL, USA, 17-20 September 2007 [Abstract G-1720b, 19 September 2007].

13 Gardasil Product Monograph. 8 May 2008. http://www.merck frosst.ca/assets/en/pdf/products/GARDASIL_1127-a_3_08E.pdf [Accessed 22 August 2008]

14 Luna J, Saah A, Hood S, Barr E. Safety, efficacy and immunogenicity of quadrivalent HPV vaccine (Gardasil) in women aged 24-45. Oral presentation at the 24th International Papillomavirus Congress, 3-9 November 2007, Beijing, China [Abstract PA1-04].

15 Smith JS, Lindsay L, Hoots B, Keys J, Franceschi S, Winer R, et al. Human papillomavirus type distribution in invasive cervical cancer and high-grade cervical lesions: a metaanalysis update. Int $J$ Cancer 2007; 121: 621-632.

\section{FACULTY OF SEXUAL \& REPRODUCTIVE HEALTHCARE MEMBERSHIP EXAMINATION}

The Membership Examination (MFSRH) consists of:

\section{$\square$ Part 1 Multiple Choice Question paper (MCQ)}

This 11/2-hour paper consists of 60 clinical science and applied science questions.

The London-based examination will be on Wednesday 22 April 2009 (the Faculty must receive applications by 1 January 2009) and on Friday 16 October 2009 (the Faculty must receive applications by 1 July 2009). The application form and information on the Part 1 can be obtained from the Faculty of Sexual and Reproductive Healthcare (FSRH) website (www.fsrh.org).

\section{Evidence Based Commentary (EBC)}

The Evidence Based Commentary replaces the case reports/dissertation component of the Membership examination and candidates can now view the first annually released topic on the Faculty website. Candidates have an absolute deadline of 31 August the following year to submit the Commentary on this topic. The Commentary must be a minimum of 1000 words and a maximum of 2000 words, excluding references, tables and appendices, and the format must follow the guidance notes. Candidates can find detailed information in the Candidate Guidance Notes for Evidence Based Commentary and the Membership Examination Regulations (September 2008) on the Faculty website.

\section{$\square$ Part 2 Examination (CRQ, SAQ, OSCE)}

This all-day examination consists of:

- Critical Reading Question examination paper (CRQ)

- Short Answer Question examination paper (SAQ)

- Objective Structured Clinical Examination (OSCE)

The Faculty must receive applications for the Part 2 to be held on Thursday 4 June 2009 by 3 January 2009. Information on the Part 2 examination, the Examination Regulations and the application form appear on the Faculty website.

The qualification is subject to re-certification every 5 years.

For the current MFSRH Examination Regulations (September 2008), information on all components of the MFSRH examination and application forms, please visit the FSRH website: www.fsrh.org (see Training \& Exams and Membership Exam) or e-mail Denise Pickford at denise@fsrh.org. Examinations, Faculty of Sexual and Reproductive Healthcare of the Royal College of Obstetricians and Gynaecologists, 27 Sussex Place, Regent's Park, London NW1 4RG, UK. Tel: +44 (0) 207724 5629. Fax: +44 (0) 2077235333. 